\title{
Contribution of Radiologist in the Management of Pseudo-aneurysm and Neck Hemorrhages in COVID-19 patients.
}

\author{
Norbert Zhang ${ }^{1}$, Jerome Lechien ${ }^{2}$, Valeria Martinez ${ }^{1}$, Robert Carlier ${ }^{1}$, and Mostafa El \\ Hajjam $^{1}$ \\ ${ }^{1} \mathrm{APHP}$ \\ ${ }^{2}$ Hospital Foch
}

November 5, 2020

\section{Key points:}

-Patients with severe coronavirus disease 2019 may have neck endothelial inflammation, pseudo-aneurysm and an increased risk of bleeding, especially during neck surgical procedures.

- The management of vascular lesions may be performed through interventional radiological procedures that may be useful to reduce the risk of virus aerosolization and healthcare provider contamination.

-Neck pseudo-aneurysm may be managed through percutaneous glue injection under ultrasonography guidance while active hemorrhage may be managed by endovascular embolization with coil.

\section{Introduction}

It has been reported that severe acute respiratory syndrome coronavirus 2 (SARS-CoV-2) may be associated with endothelial inflammation and an increasing risk of hemorrhage. ${ }^{1}$ Because the infection is associated with a high risk of aerosolization and related healthcare provider contamination, the treatment of vascular lesions has to be as conservative as possible. ${ }^{2}$ From March, $1^{\text {st }} 2020$ to May $28^{\text {th }} 2020,74$ patients with severe coronavirus disease 2019 (COVID-19) were hospitalized in our intensive care unit (ICU) and 35 patients benefiting from surgical tracheotomy (47\%). The realization of tracheotomy made sense in a context of pandemic and ICU overload because tracheotomy allowed the reduction of the duration of mechanical ventilation and the faster discharge of patients from the ICU. ${ }^{2}$ Among them, three individuals (8.5\%) developed severe bleeding from the superior thyroid artery. Two patients benefited from interventional radiology and one had surgical exploration and right superior thyroid artery ligation.

In this paper, we report the history and the conservative management of the two COVID-19 patients who had vascular lesion.

\section{Technical description}

Patient history

A 50-year-old male were hospitalized in ICU for a critical COVID-19. The patient required curative anticoagulant therapy for a right internal jugular thrombosis and were tracheotomized seven days (d7) after its admission to be weaned from the mechanical ventilation. Patient was discharged from the ICU at two weeks (d14) and he was transferred into a conventional hospitalization department. Seven days after the ICU discharge (d21), patient reported neck discomfort and important bleeding around the tracheotomy tube. The neck CT-scan revealed a pseudoaneurysm of the cricothyroid artery, which was closed from the tracheotomy tube in neck flexion position (Figure 1a, b). The pseudo-aneurysm was attributed to the vessel 
abrasion by the tracheotomy tube. According to the comorbidities of patient and the risk of aerosolization, an experimental percutaneous endovascular intervention was proposed to patient.

\section{Pseudo-aneurysm embolization}

1. The pseudoaneurysm was located with neck Doppler-Ultrasonography using a linear probe.

2. After local anaesthesia (Lidocaine hydrochloride $10 \mathrm{mg} / \mathrm{ml}$, Aguettant, Lyon, France), a fine needle (Spinocan, 0.90x88 mm, 20G, B.Braun Medical, Melsungen, Germany) was inserted until the middle of the pseudoaneurysm and radiologist injected one millilitre of an emulsion of Lipiodol $\mathbb{R}$ Ultra Fluide (LUF) (Guerbet, Aulnay-sous-Bois, France) and Glubranß2 (GEM, Viareggio, Italy) in a volume ratio of 1 to 1 pushed by $1 \mathrm{ml}$ of Glucose serum (Figure 1c, d).

3. The post-embolization tomodensitometry confirmed the emulsion deposit within the pseudo-aneurysm (Figure 1e, f). No further bleeding was reported and patient was discharged a few days later.

\section{Patient history}

A 76-year-old COVID-19 male with history of coronary stenting, type 2 diabetes, hypertension and heart arrhythmia was admitted in the ICU for respiratory distress requiring mechanical ventilation. As recommended in our ICU department, patient was tracheotomized 7 days after its ICU hospitalization. Surgical tracheotomy was performed without difficulty. Forty-eight hours post-tracheotomy (d9), patient benefited from neck CT-scan for peri-tracheal bleeding. The tomodensitometry confirmed lateral neck hematoma in front of the left superior thyroid artery (Figure 2a, b). According to the bleeding risk and the clinical condition of the patient, physicians proposed a conservative management of the hemorrhage.

\section{Hemorrhage management}

1. Patient was transferred in the radiology department. The procedure was performed through a fluoroscopy system (Multidiagnost Eleva, Philips Medical System, Best, The Netherlands).

2. Radiologist identified the left external carotid artery through right groin site puncture.

3. The culprit left superior thyroid artery was cannulated with a 0.018-inch microcatheter (Maestro 2.4 French; Merit Medical, South Jordan, UT). Angiogram demonstrated an irregular vessel.

4. The vessel was occluded with a pushable coil MicroNester@ $0.2 \times 3 \mathrm{~cm}$ (Cook Medical, Bloomington, Indiana, USA) (Figure $2 \mathrm{c}, \mathrm{d}$ ).

5. The bleeding subsequently resolved. There was no recurrence of the hemorrhage but the patient died 5 days later (d14) due to multivisceral organ failure and septic collapse.

\section{Discussion}

The surgical management of vascular complications of COVID-19 patients is challenging regarding the critical condition of many COVID-19 patients and the high-risk of aerosolization and related healthcare provider contamination. Two original findings are reported in this technical paper.

First, the occurrence of head and neck vascular complications in ICU patients, which were poorly referenced in the literature. SARS-CoV-2 is known to be associated with endothelial inflammation that weakens the vascular wall. ${ }^{1}$ In our first reported case, it seems conceivable that the tracheotomy tube could have weaken the cricoarytenoid artery through a friction mechanism. This kind of post-tracheotomy complication was however poorly reported in the literature, ${ }^{3}$ strengthening the need to monitor patients with severe COVID19 after the realization of surgical tracheotomy. In the second cases, the vascular lesion was relatively far from the anatomical site of tracheotomies that were performed in front of the third tracheal ring. Thus, we believe that the hemorrhage of the second patient was not associated with the tracheotomy procedure, but it was probably enhanced by the COVID-19 endothelial weaken, the patient comorbidities and the use of preventive anticoagulation therapy.

Second, the rational to perform conservative treatment in COVID-19 patients was based on the critical condition of patients, the high risk of aerosolization ${ }^{2,4}$ and the related risk of healthcare provider contamination, especially otolaryngologists. ${ }^{5}$ In this paper, we report two original interventional radiological approaches for 
the treatment of vascular lesions of the head and neck region. To the best of our knowledge, there is no similar paper in the literature reporting such percutaneous and endovascular approaches in the COVID-19 context. On the one hand, these approaches avoid the risk of aerosolization during the surgery revision where otolaryngologists mobilize the tracheotomy tube. On the other hand, general anesthesia is not required that may be valuable for patients who have comorbidities and critical clinical condition. Notwithstanding the COVID-19 situation, interventional radiological management of bleeding from superior or inferior thyroid arteries was rarely reported ${ }^{3}$ despite safety, fast realization and adequate post-intervention outcomes of these approaches. ${ }^{6}$

\section{Conclusion}

COVID-19 patients may have a higher risk of vascular impairments, leading to pseudo-aneurysm or head and neck hemorrhage. According to the risk of aerosolization during any head and neck surgical approach, physicians may propose conservative endovascular treatment that are rapidly performed by interventional radiologists and associated with adequate outcomes.

\section{Acknowledgments: -.}

Data availability : Data are available on request to the corresponding author.

\section{References}

1. Topal G, Loesch A, Dashwood MR. COVID-19 - Endothelial Axis and Coronary Artery Bypass Graft Patency: a Target for Therapeutic Intervention? Braz J Cardiovasc Surg . 2020 Oct 1;35(5):757-763. doi: 10.21470/1678-9741-2020-0303.

2. Chiesa-Estomba CM, Lechien JR, Calvo-Henríquez C, Fakhry N, Karkos PD, Peer S, Sistiaga-Suarez JA, Gónzalez-García JA, Cammaroto G, Mayo-Yánez M, Parente-Arias P, Saussez S, Ayad T. Systematic review of international guidelines for tracheostomy in COVID-19patients. Oral Oncol . 2020; 108:104844. doi: 10.1016/j.oraloncology.2020.104844.

3. Lin HW, Reilly BK. Pseudoaneurysm of the superior thyroid artery following revision tracheostomy. Laryngoscope . 2012; 122(7):1641-3. doi: 10.1002/lary.23291.

4. Jacob T, Walker A, Mantelakis A, Gibbins N, Keane O. A framework for open tracheostomy in COVID19 patients. Clin Otolaryngol . 2020; 45(4):649-651. doi: 10.1111/coa.13549.

5. Tysome JR, Bhutta MF. COVID-19: Protecting our ENT Workforce. Clin Otolaryngol . 2020; 45(3):311-312. doi: 10.1111/coa.13542.

6. Lee SH, Choi HJ, Yang JS, Cho YJ. Coil embolization in ruptured inferior thyroid artery aneurysm with active bleeding.J Korean Neurosurg Soc . 2014; 56(4):353-5. doi: 10.3340/jkns.2014.56.4.353.

Figure 1: Findings of patient with pseudo-aneurysm.

Pre-embolization angio-CT-scan in axial (a) and sagittal (b) views reported pseudoaneurysm close to the tracheotomy tube. The percutaneous embolization procedure was performed through a fine needle that was introduced until the center of the pseudoaneurysm and the radiologist injected emulsion material (Glue + LUF) (c). The pseudoaneurysm was occluded with the emulsion material (d). Immediate post-embolization neck CT-scan in axial (e) and sagittal (f) views showed a spot of the emulsion material (Glue + LUF) within the pseudoaneurysm (arrow).

\section{Figure 2: Findings of patient with neck hemorrhage.}

Pre-embolization angio-CT in frontal (a) and sagittal (b) views revealed a massive deep neck leakage of contrast material from the left superior thyroid artery (arrows). The imaging of embolization procedure showed left common carotid angiogram (c) and microcatheterization and coiling of the left superior thyroid $\operatorname{artery}(\mathrm{d}$, arrow). 

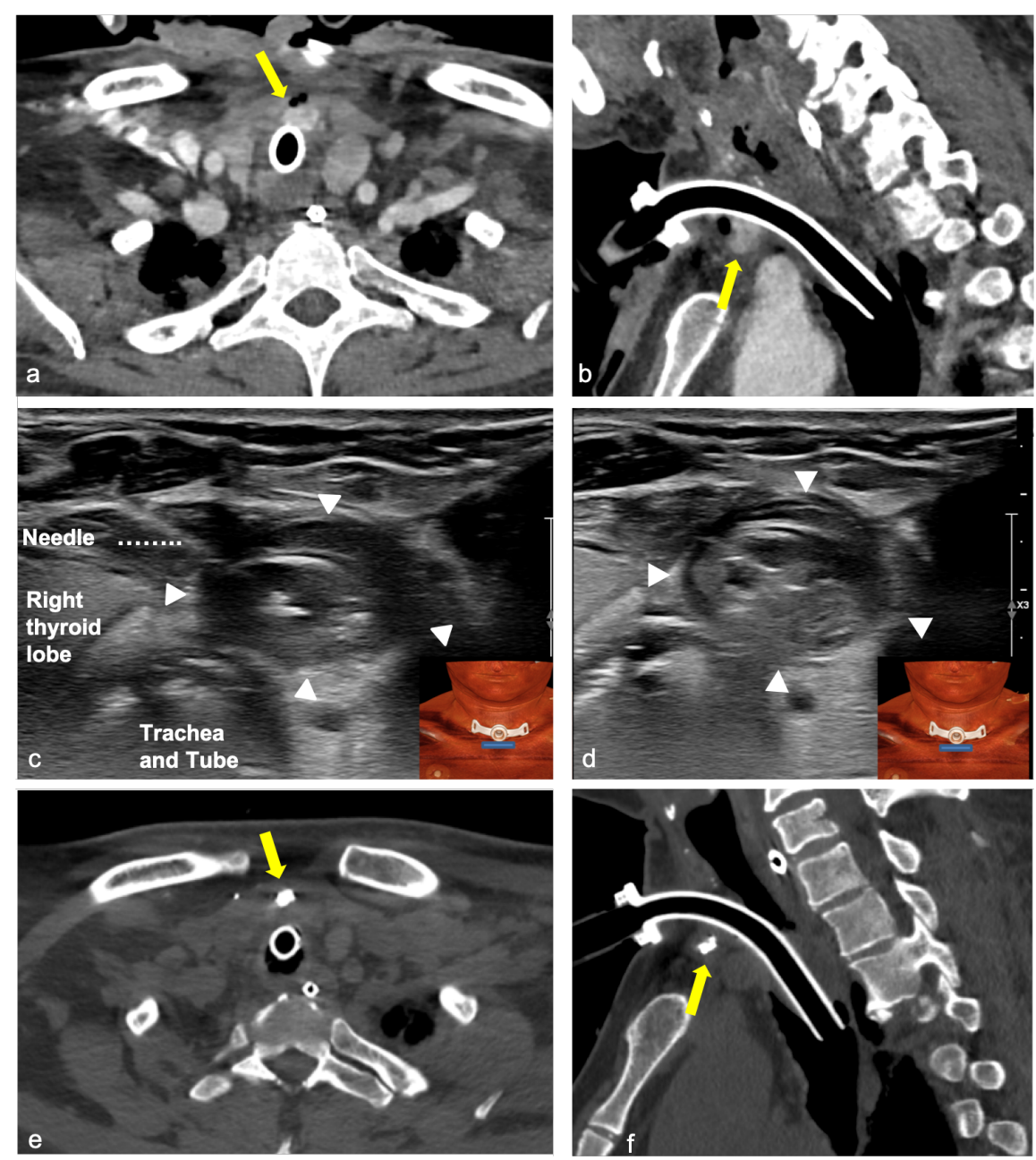


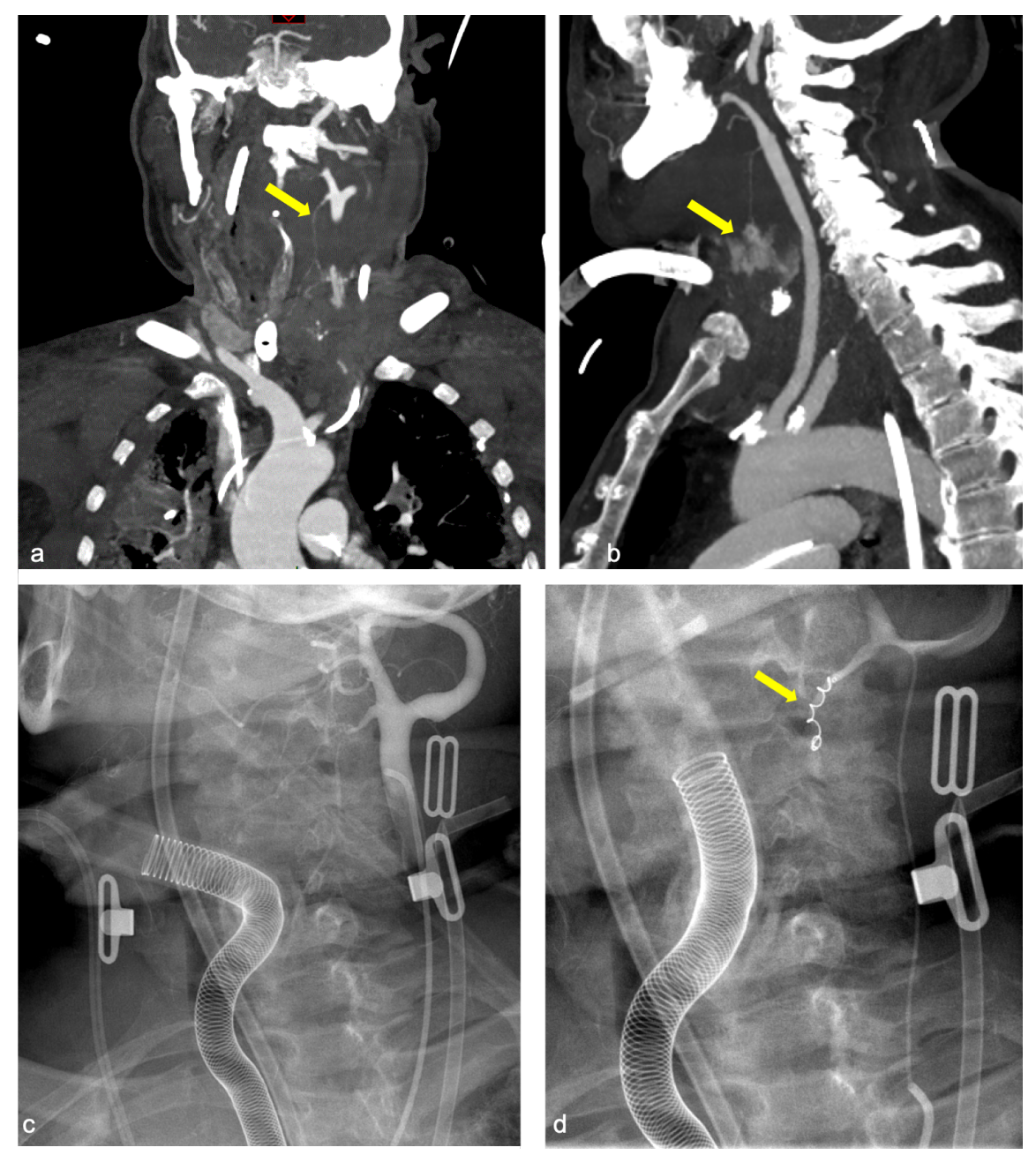

